\title{
Cotyledonoid Leiomyoma of Uterus
}

\author{
Meena L. N. • Aggarwal Abhishek · Jain Sanchit
}

Received: 6 September 2011 / Accepted: 14 June 2012/Published online: 17 August 2012

(C) Federation of Obstetric \& Gynecological Societies of India 2012

\section{Introduction}

Cotyledonoid leiomyoma is very rarely described variant of benign uterine leiomyoma. Roth et al. [1] reported a series of four cases of leiomyoma characterized by alarming extra uterine growth with a dissecting myometrial component. Because of its striking resemblance to placenta, the term cotyledonoid dissecting leiomyoma was coined for this peculiar variant. It is also called Sternberg Tumor in recognition of contribution by the late William H. Sternberg.

\section{Case Report}

A 40-year-old premenopausal woman presented with complaints of pain and lump in lower abdomen during the preceding since 8 months. On per abdomen examination, a lump of around $10 \times 12 \mathrm{~cm}$ was noticed in hypogastrium and right iliac fossa.

CA-125 was $66 \mathrm{IU} / \mathrm{ml}$. USG abdomen showed a right adnexal soft tissue mass of $10 \times 8 \mathrm{~cm}$ showing increased vascularity (Fig. 1).
Patient was explored by lower midline incision and the following findings were noted. There was a red brown tumor mass of $13 \times 10 \mathrm{~cm}$ arising from fundus of uterus with large exophytic component, which was extending into myometrium. It consisted of multiple grape-like nodules which were wrapped by anastomosing network of blood vessels, reminding of cotyledons of placenta. Bilateral ovaries and fallopian tubes were normal. Total abdominal hysterectomy with bilateral salpingo oophorectomy was done. Post-operative period was uneventful and patient was discharged on day 5 .

Histopathological report: on macroscopic examination there was a growth measuring $14 \times 7 \mathrm{~cm}$ involving the endometrial cavity and extending to fundus and forming a papillary and nodular mass on surface. Microscopic examination revealed a neoplasm comprising whorls and interlacing fascicles of smooth muscle cells. It is dissecting into adjacent myometrium in form of nodules. No atypia, mitotic activity or coagulative necrosis was seen. Marker study showed tumor cells were strongly positive for vimentin and SMA(smooth muscle actin) and were negative for S-100, CD34, and CD117 (Fig. 2).

\section{Discussion}

Cotyledonoid leiomyoma is a very rare variant of uterine leiomyoma characterized by the presence of numerous bulbous protrusions over the uterus, resembling the cotyledons of placenta. 


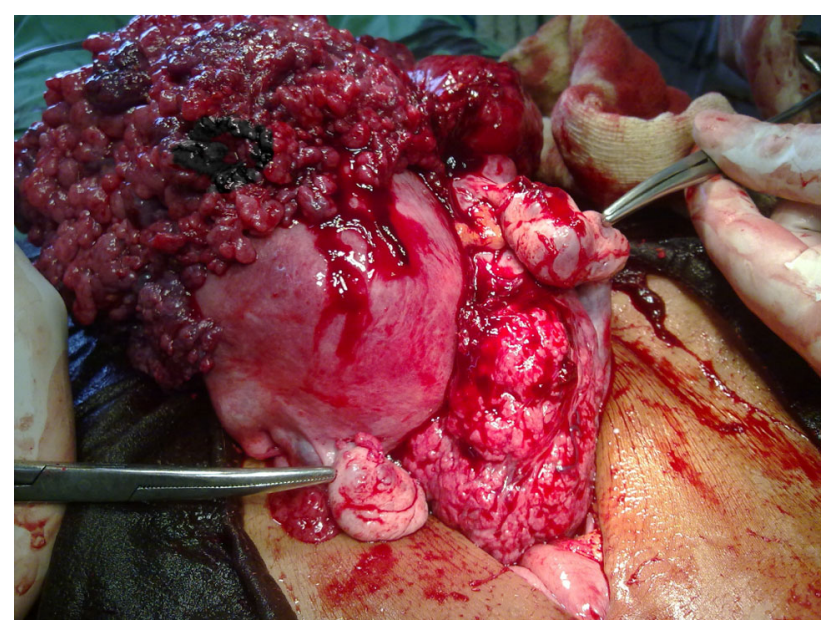

Fig. 1 Tumor mass arising from fundus of uterus and normal bilateral adnexa

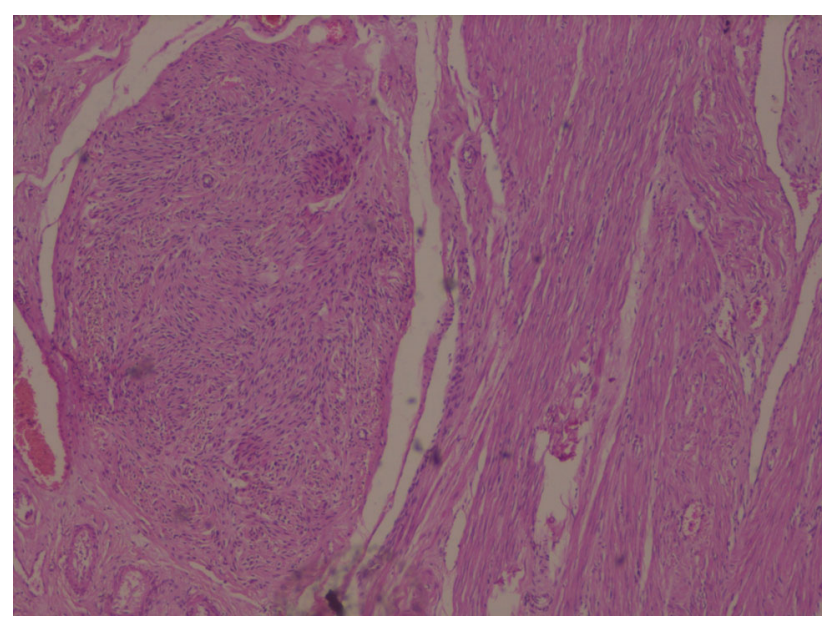

Fig. 2 Tumor is composed of interlacing fascicles of bland looking smooth muscle cells. Coagulative necrosis, mitosis and nuclear atypia are absent
The ages of patients with cotyledonoid leiomyoma ranged from 23 to 65 years [2]. The most common presenting symptoms were abdominal pain, abnormal uterine bleeding, and pelvic mass. The malignant appearance of the tumor can be attributed to lack of familiarity with alarming fungating appearance, the large size (all reported cases were $10 \mathrm{~cm}$ or larger in maximum dimension) [2] and the apparent widespread infiltrative growth. The histological features of tumor are those of a bland looking and mitotically inactive leiomyoma, and clinical outcome has been uniformly benign.

To date, no malignant behavior or recurrence has been described in these lesions with the longest follow-up period amounting to 41 years [3].

To summarize, cotyledonoid leiomyoma of uterus is a very rare variant of uterine leiomyoma with a unique gross appearance and essentially benign nature. The most important reason to recognize this unusual variant is to have a correct diagnosis and prevent overtreatment.

\section{References}

1. Roth LM, Reed RJ, Sternberg WH. Cotyledonoid dissecting leiomyoma of the uterus: the Sternberg tumor. Am J Surg Pathol. 1996;20:1455-61.

2. Cheuk W, Chan JKC, Liu JYS. Cotyledonoid leiomyoma: a benign uterine tumor with alarming gross appearance. Arch Pathol Lab Med. 2002;126:210-3.

3. Weissferdt A, Maheshwari MB, Downey GP, Rollason Raji Ganesan TP. Cotyledonoid dissecting leiomyoma of the uterus: a case report. Diagnostic pathology. 2007;2:18. doi:10.1186/17461596-2-18. 